\title{
A Hash-Based Locator/ID Mapping Mechanism
}

\author{
Zhai chenggong, Fei xianhong, and Qiao Han
}

\begin{abstract}
With the development of Internet, the original TCP/IP architecture has faced some critical challenges from scalability, mobility, security and so on. It also hampered the development of some new network technologies such as multi-homing, traffic engineering. It is well known that one of the most important reasons is IP address semantic overloading. As a result, academy commonly considered that we should give a clean-slate design for the naming and addressing architecture of the future Internet. "Locator/ID Split" is one of the most important research areas. The scalable flat-labels based mapping service is the kernel of "Locator/ID Split". HLIM uses hash-based routing to provide a deterministic mapping resolution for the edge network through distributed mapping servers. HLIM satisfies the scalability of the flat-labels mapping service, and can adapt mapping nodes' dynamic join and exit.
\end{abstract}

Index Terms-"Locator/ID Split", flat-labels, mapping service, hash, scalability.

\section{INTRODUCTION}

In the current TCP/IP protocol, the IP address has double meanings in semantics. It represents both the topological position of the network node and the identifier of the node, which is called the IP address semantic overload problem (IP Overload). IP address semantic overload is not conducive to support mobility, multi host and traffic engineering and other application requirements, affecting the scalability of Internet routing and addressing, but also brings a series of security issues.

IP address confused the function bounds of Locator and Identifier: Locator PA (Provider Assigned) address, it should be distributed according to the topology of the network, which can address the polymerization characteristics, global routing; Identifier is PI (Provider Independent) address, there is no guarantee structure characteristics, it is difficult to aggregate, generally do not support global routing. Therefore, it is difficult to achieve the above two functions with a unified address unless there is a breakthrough in flat marking routing [1]. One of the trends is to introduce two address spaces to represent the node identifier and location, in order to solve the network mobility, scalability, security and other aspects of the problem, the so-called "Locator/ID Split" [2]. After the introduction of "Locator/ID Split", and the user can conveniently use the PI address to achieve multi host, traffic engineering, avoid using the PA address by address allocation (renumbering) generated by the price, such as many IP based access control strategy, the need to change. The network security can be improved by integrating routing and

Manuscript received March 14, 2018; revised May 12. 2018.

The authors are with Army Logistics University of PLA, chongqing, China (e-mail: $18162601451 @ 163 . c o m)$. addressing schemes based on "Locator/ID Split" with source address authentication techniques.

In order to achieve "Locator/ID Split", we need to introduce two sets of address space of Locator and ID, and complete the conversion between two sets of address spaces. Because ID does not support global range routing, it is necessary to map ID to its corresponding Locator in order to forward packets to remote domains. Therefore, scalable mapping service is the core of routing and addressing system based on "Locator/ID Split".

The main challenges of scalable mapping services include: how to control query / response delay, how to avoid packet loss, and how to control the scale of mapping table, etc.. There are 3 main factors that affect the scalability of mapping service: the update frequency of mapping data, the state scale of mapping service, and the mapping query delay. It is estimated that if the "Locator/ID Split" is applied in the Internet, the mapping state of the data will reach o(1010)[3], and the scale of the global mapping table that is not reasonably constructed will be enormous. In addition, the update frequency of the mapping data should be controlled to reduce the traffic of the update message and accelerate the convergence of the mapping state. The mapping data update frequency is mainly affected by the node reachable position and the change of position. The issues to be considered in mapping services include: mapping service deployment location, mapping service access mode, mapping service security issues (such as: how to resist DOS attacks).

\section{RELATED WORK}

The most straightforward and simple way to complete the mapping from ID to Locator is to use the existing DNS system to increase the mapping record of the host identifier (ID) to the IP address (Locator) in the existing DNS. DNS has a fast query speed, but the query frequency and update speed are limited, which can not meet the mobility requirements of nodes.

GSE [4] uses DNS to complete the mapping service, and the site's DNS server introduces a new resource record format, specifying the Locator part of the address. GSE assumes that the nodes of the DNS name for the fixed identity, using the usual DNS query identifies DNS mapped to the IPv6 address when an application needs to send data, to obtain the destination by querying the DNS IPv6 AAAA records, AAAA records returned contains the specified destination Routing Stuff (Locator) and ESD (ID).

SHIM6 [5] needs to map between ULID (ID) and Locator, and then find the corresponding Locator before sending the message. SHIM6 maps ULID to Locator through DNS reverse queries, creates AAAA records on Reverse Tree, and sets SRV records on reverse trees to represent static priorities 
and weights, providing support for traffic engineering.

Six/One [6] provides many address strings (Address Bunch). All addresses in the address string are just different from the routing prefix. The host can smoothly switch between different addresses of the same address string without destroying the upper level session. The host can modify a single address string and publish it using DNS.

In addition to using DNS for mapping services, HIP [7] also introduces the way of rendezvous server. The HIP collection server maps the host identifier to a set of IP addresses, forwards the first HIP messages of the two sides of the communication, and the remaining HIP messages will be transmitted directly between the two sides of the communication. If a host's IP address changes, the host will notify the collection server. The method of set service speeds up the update speed of host identification and the transmission of mapping information. The disadvantage is that the collection server needs to maintain the complete mapping database, and the host identification namespace is very large, which increases the difficulty of the implementation of the collection server.

Some schemes (TIDR [8], LISP-ALT [9]) provide mapping services for edge networks by constructing overlay network based mapping service systems. These schemes are often improved based on existing routing protocols, such as BGP and so on, to carry mapping information. Routing protocols run over overlay networks to exchange and update mapping information between mapped service nodes. The mapping service based on the routing protocol has good scalability, but the update and convergence of the mapping information is slow.

Through the establishment of the TIDR tunnel at the edge of the network to packet forwarding network traffic, customer network prefix which requires the tunnel flow provider AS can receive the notice. To this end, TIDR introduces a new transport (transitive) BGP attribute: LOCATOR, which specifically contains the IP address of tunnel destination (locator) and the tunnel encapsulation type of specific prefix, such as GRE [10] (Generic Routing Encapsulation). The prefix declared by the LOCATOR attribute will become a pure end node identifier, and inter domain routing does not require such prefixes, which will be stored in the TIB.

LISP-ALT uses the optional logical topology (overlay network) to complete the mapping service. The overlay network is built on the Internet using BGP and GRE. ALT connects through GRE, using BGP to carry reachability information of EID prefix, and announce and assemble EID prefix

DHT has the characteristics of self configuration, self maintenance, expansibility and robustness. Therefore, people consider to apply it to the mapping service suitable for "Locator/ID Split". LISP-DHT [11] is a mapping based distributed system based on DHT. Mapping information is stored on the DHT node. LISP-DHT uses the Chord algorithm to provide mapping services, forcing each node on the Chord ring to use ID as the Chord-ID. All Chord routing information associates the (ID, Locator) mapping items owned by the Chord nodes with the corresponding Chord-ID (Key) to form a key value pair.

\section{HLIM MAPPING SERVICE}

\section{A. Basic Framework}

HLIM adopts a network based solution, and uses the distributed mapping service system to complete the conversion of Identifier space and Locator space by network devices (edge routers). HLIM to ensure that the "Locator/ID Split" on the end host transparent, end host protocol stack and the application can continue to use the original address format, but just as Identifier, is no longer the global routing capability, thereby avoiding large-scale host update difficulties, and improves the utilization rate of the address space.

HLIM introduces two sets of address spaces, Identifier and Locator. In order to support the movement of the end host, the Identifier address prefix no longer has aggregation characteristics, and evolves into a flat identifier space. Each Identifier can be considered as a fixed PI address. Locator address space is a new 64 bit PA address space, each address contains topological structure characteristics, has good aggregation characteristics, support the global routing rate.

HLIM uses the "mapping + encapsulation" approach. The edge router receives a message sent by a host, according to the mapping information obtained from the HLIM, a new head contains Locator attached to the current message, is transmitted through the GRE tunnel based on routers. In the new paper, the internal header source and destination address is Identifier (IP address), the external header source and destination address is Locator. When a packet encapsulation after reaching the destination edge router, the router will unlock the message, and sends it to the destination according to Identifier.

\section{B. HLIM Basic Mapping}

The potential size of mapping data is very large, and the centralized mapping service can not meet the requirements of scalability. Therefore, we distribute the entire mapping database to several service nodes. As shown in Figure 1, HLIM adopts the distributed mapping service mechanism, and several mapping servers are distributed in the whole network range to form a logical whole, which provides the mapping information query service for the edge routers. HLIM satisfies scalability of flat identity mapping service, and can adapt to dynamic join and leave of mapping service node.

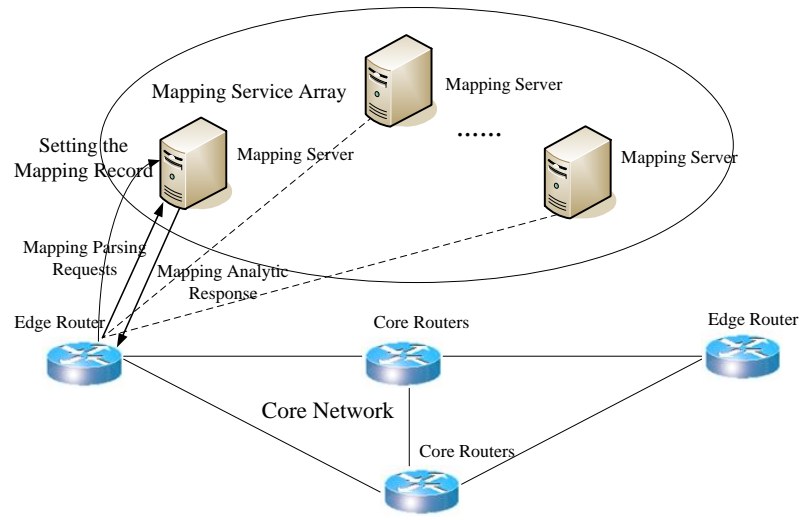

Fig. 1. HLIM mapping service system.

Because Identifier is a flat identifier space, it can not be 
aggregated, which seriously affects the scalability of the mapping service. HLIM uses hash function mapping to classify and classify the flat identity space, thus providing the guarantee for the scalability of mapping analysis. We draw on the idea of CARP (Cache Array Routing Protocol)[12] in Web proxy services, and use distributed hash mapping to provide deterministic mapping analysis for edge networks.

HLIM divides the Identifier space into several blocks by hash mapping, and distributes each block to a mapping server. This group of servers constitutes a logical whole, and maintains a complete mapping service database. The feature of HLIM is that the mapping information storage location of a specific Identifier is determined, that is, a destination Identifier is mapped to a unique mapping server.

HLIM is a single hop request parsing, the specific algorithm is as follows:

HLIM is a single hop request parsing, the specific algorithm is as follows:

- Set $h($.$) is hash function, mapping Identifier to hash$ space $\mathrm{H}$, all edge routers have the same $\mathrm{h}($.$) ;$

- assuming that the mapping service system is composed of $\mathrm{N}$ mapping servers, the hash space $\mathrm{H}$ is partitioned into $\mathrm{N}$ blocks, and each mapping server is responsible for maintaining the Locator/Identifier mapping information corresponding to a hash space $\mathrm{H}$

- when the edge router forwards the message, if there is no local Locator information corresponding to the destination Identifier, the $\mathrm{h}(\mathrm{i})(\mathrm{i}$ is the destination Identifier) is first computed, and the results obtained by $\mathrm{h}(\mathrm{i})$ calculation belong to one of the $\mathrm{N}$ blocks;

- if the $h(i)$ is a block $n$, the edge router will send the mapping resolution request to the $\mathrm{N}$ mapping server. If the mapping server has the corresponding mapping record, the mapping message is sent to the edge router through the mapping reply message.In addition to mapping resolution, edge routers are also used as data sources for mapping service systems.

Each edge router has the mapping record of the Locator/Identifier of the local end node, and uploads the record to the corresponding mapping server by using the same hash calculation method as the mapping. If the local mapping record of the edge router changes (the join of the end node, the exit, etc.), the edge router needs to update the corresponding server record.

\section{HLIM Maximum Selection Mapping}

HLIM basic mapping is only applicable to the static node structure, when a dynamic mapping server to join or leave, each mapping mapping records stored by the server will dramatically in all migration mapping server within the resulting mapping query success rate sharply decreased. This is similar to the case of CARP, where each array node is added or reduced, resulting in a change in the storage location of the $1 / 2$ record [13].

Suppose that the hash space $H$ is $[0,1]$, the mapping service system has a total of $N$ servers, that is, $H$ is divided into $N$ blocks:

$$
\left[0, \frac{1}{N}\right),\left[\frac{1}{N}, \frac{2}{N}\right),\left[\frac{2}{N}, \frac{3}{N}\right), \ldots,\left[\frac{N-1}{N}, 1\right] .
$$

After adding a mapping server, the number of nodes is $N+1$, and $H$ is divided into $N+1$ blocks:

$$
\left[0, \frac{1}{N+1}\right),\left[\frac{1}{N+1}, \frac{2}{N+1}\right),\left[\frac{2}{N+1}, \frac{3}{N+1}\right), \ldots,\left[\frac{N-1}{N+1}, \frac{N}{N+1}\right),\left[\frac{N}{N+1}, 1\right] .
$$

By (1) (2) the following easy mapping records block migration occurred:

$$
\left[\frac{1}{N+1}, \frac{1}{N}\right),\left[\frac{2}{N+1}, \frac{2}{N}\right),\left[\frac{3}{N+1}, \frac{3}{N}\right), \ldots,\left[\frac{N}{N+1}, 1\right]
$$

Accumulate the map record block of migration:

$$
\sum_{i=1}^{N} \frac{i}{N(N+1)}=\frac{1}{2} .
$$

By (4) it is known for an existing mapping service system, if $M$ mapping servers are added, the total records in the original mapping records are migrated, and the results of the mapping server are reduced. It can be seen that the mapping data migration caused by the change of the number of nodes in the mapping service system is very severe in the HLIM basic mapping.

In the HLIM basic mapping, mapping records lead to changes in node number mapping service system migration can be divided into 2 categories: one is the migration of map was recorded in the original node inside; the other is to migrate to the new node mapping records, such as mapping service system node to $N+1$ by $N$, the original mapping the record will have to migrate to the new node $1 / N+1$. We consider that the second kind of migration is rigid and unavoidable, and the first kind of migration can be avoided by accurate storage algorithm. Thus, the optimal migration ratio of HLIM is $1 / N+1$.

The HLIM maximum selection mapping algorithm is as follows:

1) each edge router has two hash functions: $h l(i)$ ( $i$ stands for destination Identifier), and $h 2(1)(1$ represents the Locator of the mapping server);

2) for a given destination Identifier, the edge router uses $h l(i)$ to compute a hash value, and uses h2(l) to compute a hash value for each mapping server of the map server list: $h 2(l 1), h 2(l 2), \ldots h 2(1 N)$;

3) $H l(i)+h 2(l)$ is calculated, and a set of values is obtained: $h l(i)+h 2(l l), h l(i)+h 2(l 2), \ldots h l(i)+h 2(l N)$ selects the largest Max $(h l(i)+h 2(l n))$ mapping server in the hash value as the storage location of the particular mapping record;

4) the edge router sends the mapping resolution request to the largest mapping server in the hash value.

Suppose the hash space $H$, the mapping service system has a total of $N$ servers, that is, $H$ is divided into $N$ blocks. For any $i$, the Maxold $(h l(i)+h 2(\ln ))$ is computed and stored on the $\mathrm{n}$ mapping server. If the number of servers is increased to $N+1$, the same $i$ is available:

$\operatorname{Maxnew}(h 1(i)+h 2(\ln ))=\operatorname{Maxold}(h l(i)+h 2(\ln ))$ or

$$
h 1(i)+h 2(l N+1)
$$

If Maxnew $(h l(i)+h 2(\ln ))=$ Maxold $(h l(i)+h 2(\ln ))$, the storage location of the record will not change, still stored on 
the $N$ mapping server; if Maxnew $(h 1(i)+h 2(\ln ))$ $=h 1(i)+h 2(l N+1)$, then the record will be migrated to the $N+1$ mapping server. In the latter case, the $N+1$ mapping server has $H / N+1$, that is, the original record of $1 / N+1$ has migrated. It is shown that the algorithm has reached the optimal value of record migration. If the number of mapped servers is increased by $\mathrm{M}$, the ratio of the migrated records is

$$
1-\prod_{i=1}^{M}\left(1-\frac{1}{N+i}\right)
$$

much smaller than $\frac{2^{M}-1}{2^{M}}$ of the HLIM basic mapping algorithm. We know that when the mapping server number, the original record is $1-\prod_{i=1}^{M}\left(1-\frac{1}{N+i}\right)$ not migrated.

(6) it can be seen that in the HLIM maximum selection mapping, the ratio of the records of migration is also related to the number of nodes in the mapping service system $\mathrm{N}$, and the larger the $\mathrm{N}$, the less the migration records. Therefore, we should ensure the size of the node appropriately when building the mapping service system.

\section{HLIM Multistage Mapping}

The above 2 algorithms still have problems in scalability: 1 ) due to the potential size of Identifier space is huge, the size of each record mapping mapping server maintenance will far exceed the current core size of routing table; 2) each edge router contains all the mapping server need to maintain accessibility (Locator) list in each query when will the list for each calculation, when the mapping server number $\mathrm{N}$ is large, the computational cost will increase. To this end, we propose HLIM multi-level mapping.

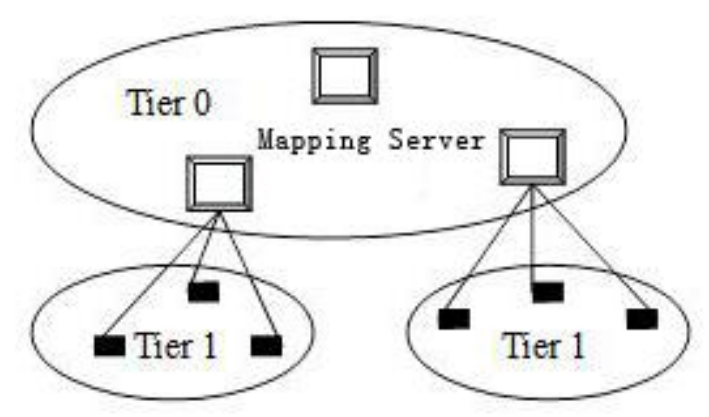

Fig. 2. HLIM multilevel mapping service array.

The whole HLIM multi-level mapping service system consists of several mapping service subsystems. Taking the 2 level mapping service array as an example, the mapping resolution request of the edge router is directly responded to 2 : Tier 0 as the root system. As needed, each node in the Tier 0 layer can re partition the hash space represented by it, thus extending the Tier 1 layer mapping system. Suppose that the hash space is $\mathrm{H}$, and that the Tier 0 layer contains $\mathrm{N}$ nodes, each node represents the hash space of $H / N$. The node $n$ of Tier 0 layer extends down the Tier 1 layer system, which contains $\mathrm{M}$ nodes, and each node of the system (including root node $n$ ) represents the hash space of H/MN.

The HLIM multi-level mapping algorithm is as follows:

- The edge router calculates the Tier 0 layer node $\mathrm{n}$ which stores the corresponding mapping records according to the mapping server list of the destination Identifier and Tier 0 layers, and sends the mapping analytic request to the node;

- The node $\mathrm{n}$ receives the request for analysis, if there is a local mapping record, message directly to the edge router mapping response; otherwise, the node $\mathrm{n}$ using a hash function to their destination Identifier and Tier 1 layer mapping server list is calculated to determine the mapping of Tier records contains 1 layers of nodes, and $\mathrm{n}$ 'request to the mapping analysis of n' edge router forwarding node;

- The node $\mathrm{n}$ 'receives the parsing request and sends the mapping reply message directly to the edge router according to the local mapping record.

HLIM multi-level mapping can greatly reduce the mapping record size maintained by each mapping server. In addition, edge routers only need to maintain a relatively small number of Tier 0 layer mapping server list, thus reducing the burden of edge routers. But it should also be seen that the HLIM multi-level mapping is no longer a single hop request parsing, increasing the forwarding path of parsing requests, thereby increasing the mapping query delay of edge routers.

\section{PERFORMANCE ANALYSIS}

The HLIM basic mapping and the maximum selection mapping are all single hop parsing, and the parsing time is $\mathrm{O}$ (1). Another mapping service scheme LISP-DHT based on Chord algorithm, because the average parsing time of the Chord algorithm is $\mathrm{O}(\log \mathrm{n})$ ( $\mathrm{n}$ is the number of nodes in the Chord ring), the mapping resolution time of the scheme is also $\mathrm{O}(\log \mathrm{n})$. We can see that the resolution efficiency of HLIM basic mapping and maximal selection mapping is better than that of LISP-DHT.

The query time of edge router (Tquery) is mainly composed of hash calculation (Process hash $\left._{\text {}}\right)$, transmission delay (delay $\left.{ }_{R S}\right)$, server query record (Process look-up $_{\text {) }}$, etc:

$$
T_{\text {query }}=\operatorname{Pr} \text { cess }_{\text {hash }}+\text { delay }_{R S}+\operatorname{Pr} \text { ocess }_{\text {look-up }} .
$$

Because the cost of hash computation and server processing is relatively fixed, we mainly consider the impact of transmission delay on query time. Suppose that the HLIM multi-level map contains the $N$ layer, and the number of mapping servers per layer is $M_{0}, M_{1}, \cdots M_{N-1}$. The probability of querying the corresponding records in the $N$ layer is $P n$ : $P_{0}=\frac{1}{M_{0} M_{1}}$

$$
\begin{aligned}
& P_{1}=\frac{1}{M_{0} M_{1} M_{2}} \quad, \ldots \quad P_{N-2}=\frac{1}{M_{0} M_{1} M_{2} \ldots M_{N-1}}, \\
& P_{N-1}=\frac{1}{M_{0} M_{1} M_{2} \ldots M_{N-2}}\left(1-\frac{1}{M_{N-1}}\right) \text {. There is a }
\end{aligned}
$$

transmission delay for HLIM multi-level mapping:

delay $_{\text {multitier-RS }}=P_{0} \cdot$ delay $_{0}+\left(1-P_{0}\right) P_{1} \cdot$ delay $_{1}+\ldots+\left(1-P_{0}\right)\left(1-P_{1}\right) \ldots\left(1-P_{N-2}\right) P_{N-1} \cdot$ delay $_{N-1} \cdot$

It is assumed that the delay of each layer is equal to delayconst, and can be obtained by (8): 


$$
\text { delay }_{\text {multi-tier-RS }}=\text { delay }_{\text {const }} \cdot \sum_{i=0}^{N-1}\left(\left(\prod_{j=0}^{i-1}\left(1-P_{j}\right)\right) \cdot P_{i}\right)
$$

The probability Pn of each layer is brought into (8) to be obtained:

delay $_{\text {multi-tier-RS }}=$ delay $_{\text {const }} \cdot\left(\sum_{i=0}^{N-2}\left(\left(\prod_{j=0}^{i-1}\left(1-\frac{1}{\prod_{k=0}^{i+1} M_{k}}\right)\right) \cdot \frac{1}{\prod_{k=0}^{i+1} M_{k}}\right)+\left(\prod_{i=0}^{N-2}\left(1-\frac{1}{\prod_{k=0}^{i+1} M_{k}}\right)\right) \cdot \frac{M_{N-1}-1}{\prod_{i=0}^{N-1} M_{i}}\right)$.

For the HLIM single stage mapping, the transmission delay is assumed to be delayconst:

$$
\frac{\text { delay }_{\text {multi-tier }-R S}}{\text { delay }_{\text {single-tier-RS }}}=\left(\sum_{i=0}^{N-2}\left(\left(\prod_{j=0}^{i-1}\left(1-\frac{1}{\prod_{k=0}^{j+1} M_{k}}\right)\right) \cdot \frac{1}{\prod_{k=0}^{i+1} M_{k}}\right)+\left(\prod_{i=0}^{N-2}\left(1-\frac{1}{\prod_{k=0}^{i+1} M_{k}}\right)\right) \cdot \frac{M_{N-1}-1}{\prod_{i=0}^{N-1} M_{i}}\right) .
$$

\section{CONCLUSION}

The core of "Locator/ID Split" is to provide scalable mapping service mechanism based on flat identifier. HLIM uses hash based single hop parsing, and provides a deterministic mapping resolution mechanism for edge networks through a distributed mapping service system. HLIM satisfies the scalability of flat mapping ID mapping service, and solves the problem of mapping record migration caused by dynamic join and leave of mapping service node to a certain extent. We also analyze the influence of multi-level mapping on query latency, and obtain the relationship between query delay and hierarchical mapping system structure. The HLIM mapping mechanism currently supports only IPv4 addresses, and next we will consider mapping queries that support both IPv4/IPv6.

\section{REFERENCES}

[1] M. Caesar, T. Condie, I. Stoica, and S. Shenker, "ROFL: Routing on Flat Labels[C]," ACM SIGCOMM'06, pp. 363-373, 2006.

[2] W. Ramirez, X. Masip-Bruin, and M. Yannuzzi, "A survey and taxonomy of ID/Locator Split Architectures[J]," Computer Networks, 2014.
[3] Y. Zhu-Liang, "Research on network architecture based on LISP protocol," Journal of Changzhou College of Information Technology, 2013.

[4] P Salmela and J Melén, "Host identity protocol proxy," in Proc. Icete-Second International Conference on E-business \& Telecommunication Networks, 2007.

[5] L. Xia and X. Ying-Jie, "A generic mobility management scheme for protocols of terminal-based identifier and locator separation in vehicular wireless networks," in Proc. the 2 nd National Conference on Information Technology and Computer Science, 2015.

[6] Z. Xiang-Ming, Z. Mei-Heng, and G. Zhong-xin, "Overview of IPv6 Transition Technology and Application Prospects," Science and Technology Horizon, 2015.

[7] R Moskowitz, "Host identity protocol architecture," Ietf-network Working Group, 2015.

[8] J. J. Adan, "Tunneled Inter-domain Routing (TIDR)," Internet Draft, 2006.

[9] A Badach, 2012, LISP+ALT-LISP Alternative Logical Topology[EB/OL]. [Online]. Available: https://www.researchgate.net/publication/281281233_LISPALT_-_LI SP_Alternative_Logical_Topology.

[10] F. Miller, A. Vandome, and J. Mcbrewster, Generic Routing Encapsulation, Alphascript Publishing, 2010.

[11] S. Brim, N. C. D. Farinacci, and V. Fuller, "LISP-CONS: A content distribution overlay network service for LISP," Internet Draft, 2008.

[12] L. Mathy, L. U. L. Iannone, and O. Bonaventure, "LISP-DHT:Towards a DHT to map identifiers onto locators," Internet Draft, 2008.

[13] K. W. Ross, "Hash routing for collections of shared web caches," IEEE Network, vol. 11, no. 6, pp. 37-44, 2011.

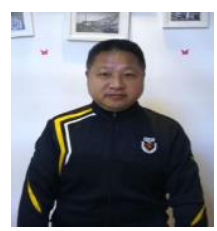

Cheng-gong zhai is a senior lecture at Army Logistics University of PLA, chongqing, China, his research field are military service information and big data.

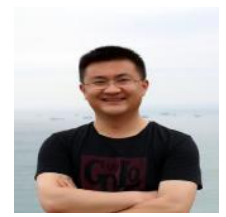

Xian-hong Fei is a full professor at Army Logistics University of PLA, chongqing, China, his research field are military service information and military education.

Han Qiao is a lecture Research Army Logistics University of PLA, chongqing, China, his field are military service information. 